\title{
Correlation of p53 and DNA repair gene mutation patterns in human malignancies indicates different tumour suppression mechanisms of $\mathbf{p 5 3}$
}

Xuemin Xue ${ }^{1}$, Lin Dong ${ }^{1}$, Liyan Xue ${ }^{1,3^{*}}$, Yong-jie Lu ${ }^{2,3^{*}}$

${ }^{1}$ Department of Pathology, National Cancer Center/National Clinical Research Center for Cancer/Cancer Hospital, Chinese Academy of Medical Sciences and Peking Union Medical College, Beijing 100021, China

${ }^{2}$ Centre for Molecular Oncology, Barts Cancer Institute, Queen Mary University of London, London, UK

${ }^{3}$ These authors contributed equally to this work

*Correspondence: xuely@cicams.ac.cn and y.j.lu@qmul.ac.uk 


\begin{abstract}
P53 suppresses tumorigenesis through multiple cellular functions/mechanisms. Recently, Janic A, et al. reported that DNA repair pathways are critical mediators of p53-dependent tumor suppression. We showed, by mining cBioPortal data of a range of human cancers, that the tendency of 'mutual exclusivity' of mutations in p53 and DNA repair genes only exist in very limited human cancer types. In the majority of human cancers, p53 mutations are equally distributed between DNA repair gene mutation positive and negative cases and in a number of human cancers, p53 and DNA repair gene mutations have a tendency of cooccurrence. These different correlation patterns of $p 53$ and DNA repair gene mutations in human malignancies may reflect different critical molecular/cellular pathways activated by p53 in different organs or cell types to suppress tumorigenesis.
\end{abstract}


P53 plays a critical role in suppressing tumor development and is inactivated by gene mutations and/or deletions in half of human cancers ${ }^{1}$. The well-established mechanisms of p53 tumor suppression are induction of cell apoptosis, cell cycle arrest and cell senescence ${ }^{1}$. However, combined loss of cell cycle suspension, apoptosis and senescence did not result in spontaneous tumorigenesis as observed upon loss of $\mathrm{p} 53^{2-4}$, indicating that there are other critical molecular/cellular mechanisms that p53 activates to suppress tumorigenesis, such as metabolic $^{2}$ and immune response ${ }^{5,6}$ pathways. Exploring the known defective molecular pathways in p53 mutated cancer cells has led to novel forms of tumor therapy strategies ${ }^{1,7}$, thus a complete illustration of all the mechanisms that p53 uses to control tumorigenesis and the specific mechanisms involved in different tumor types should improve cancer therapeutic approaches for $p 53$ mutated tumors. Recently, Janic et al. reported that DNA repair pathways were critical mediators of $\mathrm{p} 53$-dependent tumor suppression ${ }^{8}$.

DNA repair processes are critical for cells to maintain genomic stability. Deficiency in DNA repair processes, frequently caused by DNA repair gene mutations, leads to genomic instability and consequently accumulation of genomic alterations ${ }^{9}$. There are several DNA repair pathways, including mismatch repair, base-excision repair, nucleotide-excision repair, translesion synthesis, homologous recombination, non-homologous end joining, the Fanconi anemia and the O6-methylguanine DNA methyltransferase pathways ${ }^{9}$. Deficiency in mismatch repair pathways leads to microsatellite instability and consequently increased mutation burden and neo-antigen load in tumor cells, which can predict anti-PD-1/PD-L1 immunotherapy response and better than the predicting value of PD-L1 expression level ${ }^{10}$. In the paper published by Janic et al. ${ }^{8}$, the authors demonstrated in mouse models that DNA repair processes are critical mediators of p53-dependent tumor suppression as knockdown of p53 target genes implicated in DNA repair, including Mlh1, Msh2, Rnf144b, Cav1 and Ddit4, accelerated MYC-driven lymphoma development to a similar extent as knockdown of p53, although not all DNA repair genes had equal effect in tumorigenesis. To translate this 
research finding from mouse models into human cancers, they analyzed leukemia, lymphoma and colorectal cancer data in the cBioPortal and suggested that p53 and DNA repair gene mutations are mutually exclusive in those human malignancies ${ }^{8}$.

This study provides new insight into p53 tumor suppression mechanisms and would help with the development of new therapeutic approaches. For example, if p53 has a similar role to mismatch repair genes Mlh1 and Msh2, then p53 mutation, which is common in human cancers, may be used to predict response to anti-PD-1/PD-L1 immunotherapy. We analyzed cBioPortal data ${ }^{11,12}$ of a broad range of human cancers for the general applicability of this association of mutations in p53 and reported p53-targeted DNA repair genes, as described in the paper by Janic et $\mathrm{al}^{8}$. Our reanalysis using the same approach as Janic et $\mathrm{al}^{8}$ of the leukemia, lymphoma and colon cancer data produced similar distribution patterns of p53 mutation in relation to these DNA repair gene mutations ${ }^{8}$. However, apart from colorectal cancer, we did not find an inverse correlation of p53 and these DNA repair gene mutations (Supplementary Figure 1). In hematological malignancies, where the frequencies of mutations in both $p 53$ and DNA repair genes are very low (each DNA repair gene mutation rate is $<1 \%$ ), the chance of these two types of mutations co-existing in the same patient is expected to be rare. Hence, neither in the original publication (Fig S19) ${ }^{8}$ nor in our analysis (Supplementary Figure 1), is mutual exclusivity of mutations in $p 53$ and these DNA repair genes statistically significant (all $\mathrm{p}>0.4$ ). Therefore, the data cannot support mutual exclusivity of p53 and DNA repair gene mutations in hematological malignancies.

As amplification is unlikely to cause loss of function of p53 or DNA repair genes, we did further correlation analysis excluding amplification of these genes (see Supplementary Methods). We also performed the correlation analysis between mutations of $p 53$ and any of these DNA repair genes in combination to increase the statistical power compared to individual DNA repair genes. With this combination, we still did not find a significant 
inverse correlation between these two types of mutations in hematological malignancies (Table 1 and Supplementary Figure 2).

In our further analysis of other human cancers, we found that p53 mutations are equally distributed between DNA repair gene mutation positive and negative cases in many human cancer types, including prostate, ovarian, liver, head and neck, esophageal, stomach and endometrial cancers and cholangiocarnoma (Table 1 and Supplementary Figure 2). Only in lung squamous carcinoma is there a trend $(\mathrm{p}=0.158)$ of reverse correlation between $p 53$ and any of these DNA repair gene mutations with $M L H 1$ mutation being significantly $(\mathrm{p}<0.006)$ reversely correlated with $p 53$ mutation prior to multiple testing correction. Most importantly, in breast cancer, melanoma, adrenocortical carcinoma and glioma, we found that mutations in p53 and DNA repair genes are closely associated with each other and these DNA repair gene mutations have a significant $(\mathrm{p}<0.001,<0.001,0.008$ and $<0.001$ respectively) tendency of co-occurrence with p53 mutation (Table 1, Figure 1). In cervical squamous carcinoma and lung adenocarcinoma, there is a trend $(\mathrm{p}=0.119$ and 0.055 respectively) of co-occurrence of these two types of mutations, with some DNA repair gene mutations significantly $(p<0.05)$ correlated with $p 53$ mutation prior to multiple testing adjustment in cervical cancer. In these tumors where p53 and DNA repair gene mutations have a significant tendency of cooccurrence, DNA repair processes are unlikely to be the mediators of p53-dependent tumor suppression. Therefore, in human malignancies, different correlation patterns of p53 and DNA repair gene mutations can exist depending on tumor types. The difference in association of p53 and DNA repair gene mutation patterns may indicate different functions of p53 in action in human malignancies and different therapeutic strategies should be developed accordingly. DNA repair processes may only be the critical mediators of p53-dependent tumor suppression in colorectal cancer and a proportion of $p 53$ mutated lung squamous carcinoma. In human cancers where p53 and DNA repair gene mutations co-exist, p53 may play a different role in suppressing tumorigenesis, such as p53-mediated activation of cancer 
immunity, that p53 function has to be lost to enable cancer cells, with DNA repair gene mutation induced high neo-antigen, to escape the p53-mediated antitumor immunity. This is supported by the observed association between p53 mutation and lack of tumor infiltrating lymphocytes reported in the literature ${ }^{6,13}$. Further investigations of the p53 functions in these different conditions in association with DNA repair genes are warranted.

Author contributions: YJ.L, and L.X contribute to the concept of the study. X.X and L.D performed the data analysis. YJ.L drafted the manuscript text and X.X, L.D, L.X, and YJ.L wrote the final manuscript.

Competing interests: The authors declare no competing interests.

\section{References:}

1. Cheok, C.F., Verma, C.S., Baselga, J. \& Lane, D.P. Translating p53 into the clinic. Nature reviews. Clinical oncology 8, 25-37 (2011).

2. Li, T., et al. Tumor suppression in the absence of p53-mediated cell-cycle arrest, apoptosis, and senescence. Cell 149, 1269-1283 (2012).

3. Brady, C.A., et al. Distinct p53 transcriptional programs dictate acute DNA-damage responses and tumor suppression. Cell 145, 571-583 (2011).

4. Valente, L.J., et al. p53 efficiently suppresses tumor development in the complete absence of its cell-cycle inhibitory and proapoptotic effectors p21, Puma, and Noxa. Cell reports 3, 1339-1345 (2013).

5. Munoz-Fontela, C., Mandinova, A., Aaronson, S.A. \& Lee, S.W. Emerging roles of p53 and other tumour-suppressor genes in immune regulation. Nature reviews. Immunology 16, 741-750 (2016).

6. Wellenstein, M.D. \& de Visser, K.E. Cancer-Cell-Intrinsic Mechanisms Shaping the Tumor Immune Landscape. Immunity 48, 399-416 (2018). 
7. Bykov, V.J.N., Eriksson, S.E., Bianchi, J. \& Wiman, K.G. Targeting mutant p53 for efficient cancer therapy. Nature reviews. Cancer 18, 89-102 (2018).

8. Janic, A., et al. DNA repair processes are critical mediators of p53-dependent tumor suppression. Nat Med 24, 947-953 (2018).

9. Dietlein, F., Thelen, L. \& Reinhardt, H.C. Cancer-specific defects in DNA repair pathways as targets for personalized therapeutic approaches. Trends in genetics : TIG 30, 326-339 (2014).

10. Song, D., et al. Bladder cancer, a unique model to understand cancer immunity and develop immunotherapy approaches. J Pathol 249, 151-165 (2019).

11. Cerami, E., et al. The cBio cancer genomics portal: an open platform for exploring multidimensional cancer genomics data. Cancer Discov 2, 401-404 (2012).

12. Gao, J., et al. Integrative analysis of complex cancer genomics and clinical profiles using the cBioPortal. Sci Signal 6, pl1 (2013).

13. Cui, Y. \& Guo, G. Immunomodulatory Function of the Tumor Suppressor p53 in Host Immune Response and the Tumor Microenvironment. International journal of molecular sciences 17(2016).

\section{Supplementary information:}

Supplementary methods

Supplementary Figure 1

Supplementary Figure 2 
Table 1. Correlation of mutations in $p 53$ and any of the 10 p53 regulated DNA repair genes together in human tumors based on genomic sequencing data from cBioPortal ${ }^{11,12}$ datasets

\begin{tabular}{|c|c|c|c|c|}
\hline Cancer & Data & Log2_OR & Tendency & p.value \\
\hline Colorectal Adenocarcinoma & Combined study* & -1.348 & Mu-ex & $2.82 \times 10^{-6}$ \\
\hline Skin Cutaneous Melanoma & TCGA, Provisional & 2.193 & Co-oc & $6.65 \times 10^{-5}$ \\
\hline Merged Cohort of LGG and GBM & TCGA, Cell 2016 & 2.697 & Co-oc & $1.65 \times 10^{-4}$ \\
\hline Breast Invasive Carcinoma & TCGA, Provisional & 1.280 & Co-oc & $6.72 \times 10^{-4}$ \\
\hline Adrenocortical Carcinoma & TCGA, Provisional & 2.700 & Co-oc & 0.008 \\
\hline Cervical Squamous Cell Carcinoma & TCGA, PanCancer Atlas & 1.330 & - & 0.119 \\
\hline Cholangiocarcinoma & TCGA, Provisional & 0.794 & - & 0.546 \\
\hline Esophageal Adenocarcinoma & TCGA, PanCancer Atlas & s 1.397 & - & 0.302 \\
\hline Head and Neck Squamous & CellTCGA, Provisional & 0.198 & - & 0.420 \\
\hline \multicolumn{5}{|l|}{ Carcinoma } \\
\hline Haematological malignancy & Combined study* & -1.197 & - & 0.349 \\
\hline Liver Hepatocellular Carcinoma & TCGA, Provisional & 0.151 & - & 0.501 \\
\hline Lung Adenocarcinoma & TCGA, Provisional & 0.928 & - & 0.055 \\
\hline Lung Squamous Cell Carcinoma & TCGA, Provisional & -0.839 & - & 0.158 \\
\hline Ovarian Serous Cystadenocarcinoma & TCGA, Provisional & 0.021 & - & 0.621 \\
\hline Prostate Adenocarcinoma & TCGA, Provisional & 0.228 & - & 0.381 \\
\hline Gastric Adenocarcinoma & TCGA, Provisional & 0.482 & - & 0.150 \\
\hline Uterine Corpus Endometrial Carcinoma & na TCGA, Provisional & -0.069 & - & 0.546 \\
\hline
\end{tabular}

* The data we chose in our analysis is the same to those in the paper of Janic et al. ${ }^{8}$; Tendency not clear or no tendency; Co-oc: co-occurrence; Mu-ex: mutual exclusivity 
a

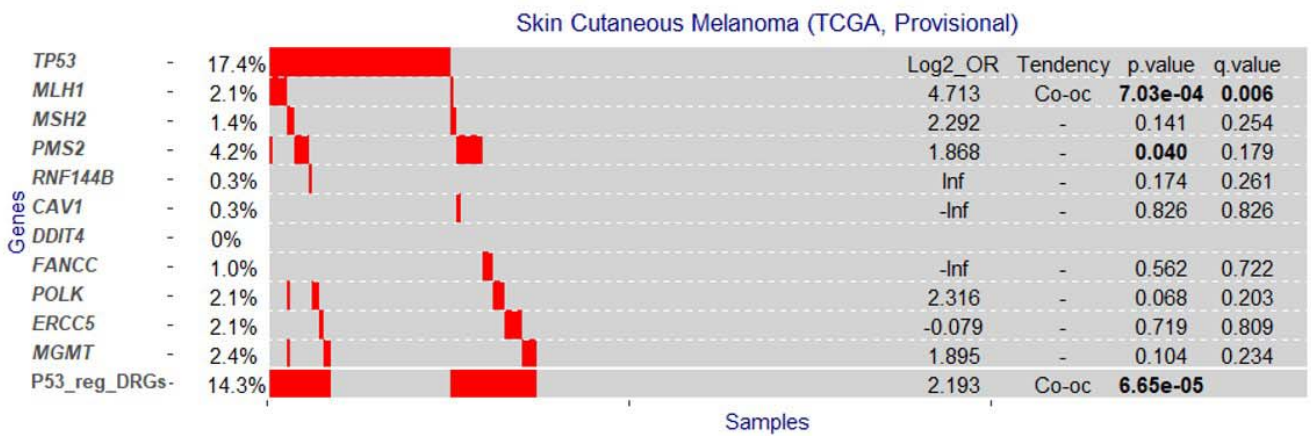

b

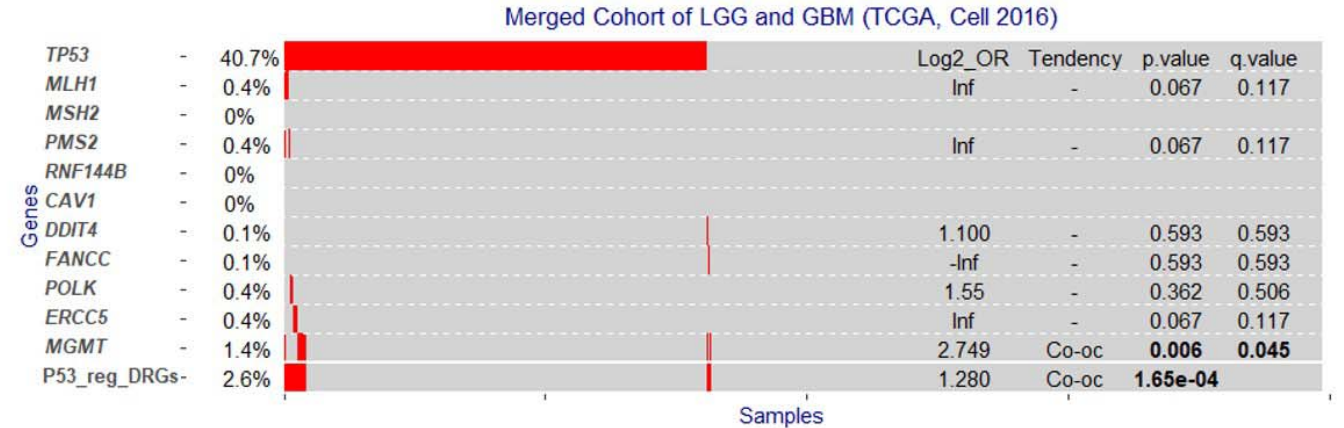

C

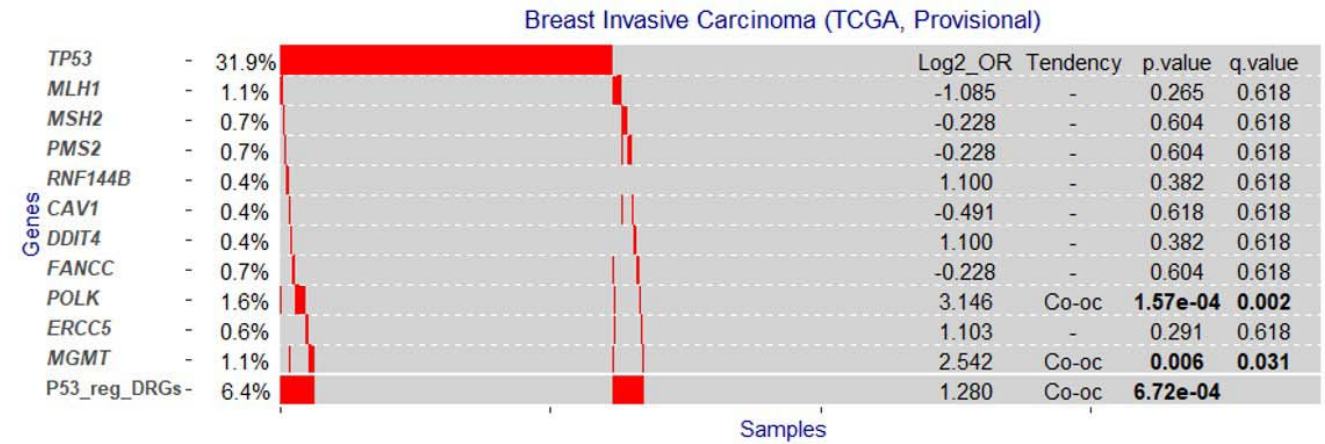

d

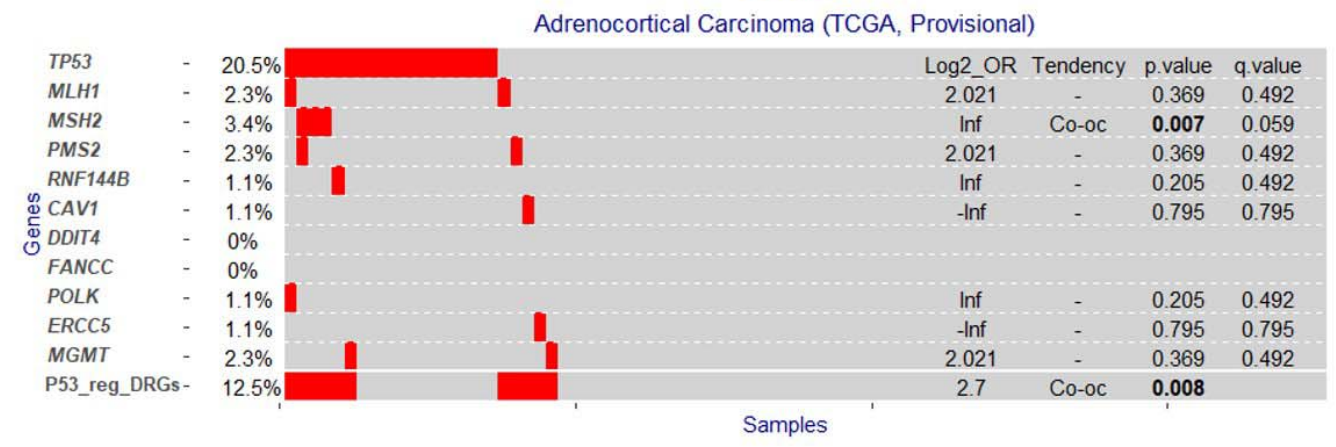

Figure 1. P53(TP53) and DNA repair gene mutation distribution in melanoma (a), glioma (b), breast invasive carcinoma (c) and Adrenocortical Carcinoma (d) based on cBioPortal 11,12 data. OR: odd ratio; Co-oc: co-occurrence; e-0n: $\mathrm{X} 10^{-\mathrm{n}}$; Inf: Infinity; P53_reg_DRGs: mutation in any of the 10 p53 regulated DNA repair genes in combination. 